\title{
Increasing the convergence rate to steady-state by using multiple penalty terms applied in a domain
}

Hannes Frenander and Jan Nordström

\section{Linköping University Post Print}

\section{Tweet}

N.B.: When citing this work, cite the original article.

Original Publication:

Hannes Frenander and Jan Nordström, Increasing the convergence rate to steady-state by using multiple penalty terms applied in a domain, 2013, AIAA Aerospace Sciences - Fluid Sciences Event.

http://dx.doi.org/10.2514/6.2013-2957

From the 21st AIAA Computational Fluid Dynamics Conference 24 - 27 June 2013 San Diego, California. 


\title{
Increasing the convergence rate to steady-state by using multiple penalty terms applied in a domain
}

\author{
Hannes Frenander* \\ Linköping University, Sweden
}

\author{
Jan Nordström ${ }^{\dagger}$ \\ Linköping University, Sweden
}

\begin{abstract}
We introduce a new weak boundary procedure for high order finite difference methods applied to the linearized Euler and Navier-Stokes equations using summation-by-parts operators. Stability is obtained by using weak boundary conditions on penalty form. We demonstrate how to add on multiple penalties in the near boundary domain such that stability is preserved and an increased speed of convergence to steady-state is obtained.
\end{abstract}

\section{Nomenclature}

\author{
Density \\ Velocity \\ Speed of sound \\ Variable value vector \\ Absolute temperature \\ Pressure \\ Adiabatic index
}

\section{Introduction}

We consider a finite difference approximation with summation-by-parts (SBP) operators and weakly imposed boundary conditions. The SBP operators are constructed to mimic integration by parts for the continuous problem. A detailed description on how these operators are constructed for first and second order derivatives can be found in the work by Strand ${ }^{1}$ and in the work by Mattson and Nordström. ${ }^{2}$ Difference operators with the summation-by-parts property and weakly imposed boundary conditions leads to energy stability $^{3}$ if well-posed boundary conditions are available. The boundary conditions are imposed weakly with the simultaneous approximation terms (SAT) ${ }^{4}$ method where the penalty parameters are chosen such that the numerical approximation becomes stable. This procedure has been extended to a penalty domain near the boundary. ${ }^{5}$ This adds more flexibility to the numerical scheme and properties such as increased convergence to steady state and less reflective boundary conditions ${ }^{5}$ can be obtained. The technique is somewhat similar to previously developed methods such as perfect matched layers (PML) ${ }^{6}$ and sponge layers. ${ }^{7}$

In this work, we consider the steady-state problem for the linearized Euler and Navier-Stokes equations with constant coefficients. Well-posed boundary conditions and stable numerical approximations will be derived for the linearized equations in one dimension. The multiple penalties technique will be applied to the numerical scheme such that stability is preserved. This is done by choosing the penalty matrices in an appropriate way. With stability guaranteed, a number of free parameters still exists. These free parameters can be chosen in order to give the numerical scheme the desired properties. The multiple penalty technique offer many possibilities, but it also puts new demands on the data of the problem since the solution must be known in the near boundary domain for accuracy reasons.

*Ph. D Student, Department of Mathematics, Linköping University, SE-581 83,Linköping, Sweden.

${ }^{\dagger}$ Professor, Department of Mathematics, Linköping University, SE-581 83,Linköping, Sweden. 


\section{Theory}

We will in this section derive well-posed boundary operators for the linearized Navier-Stokes equations. The linearized Euler equations with well-posed boundary conditions is obtained by letting the viscosity $\epsilon \rightarrow 0$ in the Navier-Stokes equations. The standard SBP-SAT technique will be applied to the semi-discrete form of the problem and the penalty operators are chosen in order to obtain stability. Finally, we will show how the multiple penalties are applied in the penalty domain such that stability is preserved in the process.

\section{A. The linearized Navier-Stokes equation}

We consider the linearized and symmetrized ${ }^{8}$ Navier-Stokes equations in one dimension

$$
u_{t}+A u_{x}=\epsilon C u_{x x}
$$

with

$$
A=\left[\begin{array}{ccc}
\bar{v} & \frac{\bar{c}}{\gamma} & 0 \\
\overline{\bar{c}} & \bar{v} & \bar{c} \sqrt{\frac{\gamma-1}{\gamma}} \\
0 & \bar{c} \sqrt{\frac{\gamma-1}{\gamma}} & \bar{v}
\end{array}\right], C=\left[\begin{array}{ccc}
0 & 0 & 0 \\
0 & \alpha & 0 \\
0 & 0 & \beta
\end{array}\right]
$$

where $\alpha=\frac{\bar{\lambda}+2 \bar{\mu}}{\bar{\rho}}, \beta=\frac{\gamma \bar{k}}{\operatorname{Pr} \bar{\rho}}, \bar{\lambda}$ is the coefficient of heat conduction, $\bar{\mu}$ the shear coefficient, $\operatorname{Pr}$ the Prandtl number, $\bar{k}$ the coefficient of heat conduction and $\epsilon$ the ratio between the Mach and Reynolds number. The mean velocity, density, temperature and speed of sound are written $\bar{v}, \bar{\rho}, \bar{T}$ and $\bar{c}$, respectively. The overbar denotes a reference state. The unknowns are

$$
u=\left[\frac{\bar{c}}{\bar{\rho} \sqrt{\gamma}} \rho, v, \frac{1}{\bar{c} \sqrt{\gamma(\gamma-1)}} T\right]^{T}
$$

with all components linearized and normalized according to $\rho=\frac{\rho}{\rho_{\infty}} T=\frac{T}{T_{\infty}} p=\frac{p}{p_{\infty}\left(c_{\infty}\right)^{2}}$ where the $\infty$ index denotes a free stream value. In the rest of this paper, we omit the constants in front of $\rho$ and $T$ by letting $\frac{\bar{c}}{\bar{\rho} \sqrt{\gamma}} \rho \rightarrow \rho$ and $\frac{1}{\bar{c} \sqrt{\gamma(\gamma-1)}} T \rightarrow T$.

Well-posed boundary conditions are established by multiplying (1) with $u^{T}$ from the left and integrate over the domain $x \in[0,1]$

$$
\|u\|_{t}^{2}+\left[u^{T} A u-2 \epsilon u^{T} C u_{x}\right]_{0}^{1}=-2 \epsilon \int_{0}^{1} u_{x}^{T} C u_{x} d x
$$

where $C=C^{T} \geq 0$. For bounded energy, the second term on the left-hand side of (2) must be non-negative. At the inflow boundary, we use the boundary conditions derived by Lindström and Nordström ${ }^{9}$

$$
\begin{aligned}
-\sqrt{\frac{\gamma-1}{\gamma} \rho+\frac{1}{\sqrt{\gamma}}} T & =g_{1} \\
\frac{1}{2 \sqrt{\gamma}} \rho+\frac{1}{\sqrt{2}} v+\sqrt{\frac{\gamma-1}{2 \gamma}} T & =g_{2} \\
\epsilon \alpha v_{x}-\epsilon \sqrt{\frac{\gamma-1}{\gamma}} \beta T_{x} & =g_{3}
\end{aligned}
$$

where $g_{1,2,3}$ are given boundary data. The first two boundary conditions are actually the characteristic variables of the problem, related to positive eigenvalues of $A$.

We will now derive well-posed boundary conditions at the outflow boundary by considering the indefinite part of (2) at $x=1$

$$
\|u\|_{t}^{2}+u^{T} A u-2 \epsilon u^{T} C u_{x} \leq 0
$$

where the right-hand side of (2) and the boundary terms at $x=0$ have been neglected. Bounded energy and well-posedness is ensured if

$$
u^{T} A u-2 \epsilon u^{T} C u_{x} \geq 0 .
$$


By expanding (4), one arrives at

$$
\bar{v} \rho^{2}+2 \frac{c}{\sqrt{\gamma}} v \rho+\bar{v} v^{2}+2 \bar{c} \sqrt{\frac{\gamma-1}{\gamma}} v T+\bar{v} T^{2}-2 \epsilon \alpha v v_{x}-2 \epsilon \beta T T_{x} \geq 0 .
$$

We introduce the boundary conditions

$$
\begin{aligned}
\epsilon T_{x} & =f_{1} \\
\delta \rho+\eta T-\epsilon \alpha v_{x} & =f_{2}
\end{aligned}
$$

where $\delta, \eta$ are constants to be determined and $f_{1,2}$ are the given boundary data. Using (6) with zero boundary data in relation (5) yields the condition

$$
\bar{v} \rho^{2}+2\left(\frac{c}{\sqrt{\gamma}}-\delta\right) v \rho+\bar{v} v^{2}+2\left(\bar{c} \sqrt{\frac{\gamma-1}{\gamma}}-\eta\right) v T+\bar{v} T^{2} \geq 0
$$

This paper focus on subsonic flows with $0<\bar{v}<\bar{c}$, so the problem is made well-posed by canceling the indefinite terms by

$$
\delta=\frac{\bar{c}}{\sqrt{\gamma}}, \quad \eta=\bar{c} \sqrt{\frac{\gamma-1}{\gamma}} .
$$

The final outflow boundary conditions in (6) becomes

$$
\begin{aligned}
\epsilon T_{x} & =f_{1} \\
\frac{\bar{c}}{\sqrt{\gamma}} \rho+\bar{c} \sqrt{\frac{\gamma-1}{\gamma}} T-\epsilon \alpha v_{x} & =f_{2} .
\end{aligned}
$$

Note that the second equation in (7) can be written $\frac{p}{\bar{\rho}}-\epsilon \alpha v_{x}=f_{2}$. Together with the boundary conditions at the inflow boundary, given by (3), and at the outflow boundary, given by (7), the problem (1) is well-posed.

\section{Semi-discrete formulation}

The standard SBP-SAT discretization of the problem (1) is

$$
\begin{aligned}
u_{t}+\left(D_{1} \otimes A\right) u & =\epsilon\left(D_{2} \otimes C\right) u \\
& +\left(P^{-1} E_{0} \otimes \Sigma_{0}\right)\left(X^{T} u-\bar{g}_{0}\right) \\
& +\left(P^{-1} E_{0} \otimes \Sigma_{1}\right)\left(K\left(D_{1} u\right)_{0}-\bar{g}_{3}\right) \\
& +\left(P^{-1} D_{1}^{T} E_{0} \otimes \Sigma_{2}\right)\left(L u_{0}-\tilde{g}\right) \\
& +\left(P^{-1} E_{N} \otimes \Sigma_{3}\right)\left(F u_{N}-\epsilon \alpha G\left(D_{1} u\right)_{N}-\bar{f}_{2}\right) \\
& +\left(P^{-1} E_{N} \otimes \Sigma_{4}\right)\left(\epsilon H\left(D_{1} u\right)_{N}-\bar{f}_{1}\right)
\end{aligned}
$$

where

$F=\left[\begin{array}{ccc}\overline{\bar{c}} & 0 & \sqrt{\frac{\gamma-1}{\gamma}} \bar{c} \\ 0 & 0 & 0 \\ 0 & 0 & 0\end{array}\right], G=\left[\begin{array}{ccc}0 & 1 & 0 \\ 0 & 0 & 0 \\ 0 & 0 & 0\end{array}\right], H=\left[\begin{array}{ccc}0 & 0 & 0 \\ 0 & 0 & 0 \\ 0 & 0 & 1\end{array}\right], K=\left[\begin{array}{ccc}0 & \alpha & -\sqrt{\frac{\gamma-1}{\gamma}} \beta \\ 0 & 0 & 0 \\ 0 & 0 & 0\end{array}\right], L=\left[\begin{array}{ccc}0 & \sqrt{\frac{\gamma-1}{\gamma}} \bar{c} & \bar{c} \\ 0 & 0 & 0 \\ 0 & 0 & 0\end{array}\right]$.

We have used the notation $D_{1}=P^{-1} Q, D_{2}=D_{1}^{2}, \overline{g_{0}}=\left[g_{1}, g_{2}, g_{3}\right]^{T}, \overline{g_{3}}=\left[g_{3}, 0,0\right]^{T}, \overline{f_{1}}=\left[0,0, f_{1}\right]^{T}$, $\bar{f}_{2}=\left[f_{2}, 0,0\right]^{T}$ and $\tilde{g}=\left[\frac{\bar{c} / \sqrt{\gamma} g_{0}+\sqrt{2(\gamma-1) / \gamma} g_{1}}{\sqrt{2} \bar{c}}, 0,0\right]^{T}$. The matrix $X$ contains the eigenvectors of $A$, such that $A=X \Lambda X^{T}$ and $E_{0, N}$ are matrices that projects $u$ on the first and last grid point, respectively. The penalty matrices $\Sigma_{0,1,2}$ are derived by Lindström and Nordström. ${ }^{9}$

We will now derive $\Sigma_{3,4}$ such that the approximation becomes stable at the outflow boundary. By multiplying (8) with $u^{T}\left(P \otimes I_{3}\right)$ from the left, disregarding the terms from the left boundary and letting all data be zero, we obtain

$$
\|u\|_{t}^{2} \leq-u_{N}^{T} A u_{N}+2 \epsilon u_{N}^{T} C u_{N x}+2 u_{N}^{T} \Sigma_{3}\left(F u_{N}-\epsilon \alpha G u_{N x}\right)+2 \epsilon u_{N}^{T} \Sigma_{4} H u_{N x}-2 \epsilon u_{x}^{T}(P \otimes C) u_{x} .
$$


In (9) we used the abbreviation $u_{x}=D_{1} u$ and the norm $\|u\|^{2}=u^{T}\left(P \otimes I_{3}\right) u$. The last term on the righthand side of (9) is negative since $P$ is positive definite and $C$ is positive semi definite, so it can safely be neglected. To ensure bounded energy and stability, we study the inviscid and viscous terms separately and demand

$$
\begin{gathered}
-u_{N}^{T} A u_{N}+2 u_{N}^{T} \Sigma_{3} F u_{N} \leq 0 \\
2 \epsilon u_{N}^{T} C u_{N x}-2 \epsilon \alpha u_{N}^{T} \Sigma_{3} G u_{N x}+2 \epsilon u_{N}^{T} \Sigma_{4} H u_{N x} \leq 0 .
\end{gathered}
$$

The inequalities (10) and (11) are sufficient conditions for stability at the outflow boundary. We make the ansatz

$$
\Sigma_{3}=\left[\begin{array}{ccc}
0 & 0 & 0 \\
\delta_{1} & 0 & 0 \\
0 & 0 & 0
\end{array}\right], \quad \Sigma_{4}=\left[\begin{array}{ccc}
0 & 0 & 0 \\
0 & 0 & 0 \\
0 & 0 & \delta_{2}
\end{array}\right]
$$

where $\delta_{1,2}$ are constants to be determined. Equation (11) can now be written as

$$
2 \epsilon \alpha v_{N} v_{N x}+2 \epsilon \beta T_{N} T_{N x}-2 \epsilon \alpha \delta_{1} v_{N} v_{N x}+2 \epsilon \delta_{2} T_{N} T_{N x} \leq 0
$$

The indefinite terms are canceled out by $\delta_{1}=1$ and $\delta_{2}=-\beta$. With these values of $\delta_{1,2}$, condition (10) can be written

$$
u_{N}^{T} A u_{N}-2 u_{N}^{T} \Sigma_{3} F u_{N}=\bar{v} \rho^{2}+\bar{v} v^{2}+\bar{v} T^{2} \geq 0 .
$$

Note that with the chosen values of $\delta_{1,2}$, the relation (10) is always satisfied and stability is obtained. The final form of the penalty matrices is then

$$
\Sigma_{3}=\left[\begin{array}{lll}
0 & 0 & 0 \\
1 & 0 & 0 \\
0 & 0 & 0
\end{array}\right], \quad \Sigma_{4}=\left[\begin{array}{ccc}
0 & 0 & 0 \\
0 & 0 & 0 \\
0 & 0 & -\beta
\end{array}\right] .
$$

With the penalty matrices given by (12) together with the matrices $\Sigma_{0,1,2}$ derived by Lindström and Nordström, ${ }^{9}$ the numerical scheme in $(8)$ is stable.

\section{Multiple penalties}

In this paper, we focus on a domain close to the boundaries where multiple penalties can be applied. Let $\left(P^{-1} \otimes I_{3}\right) \tilde{Q}$ be the total operator in (8), including both the space-discretization and the standard penalty terms with zero boundary data; the multiple penalties are then applied according to

$$
u_{t}+\left(P^{-1} \otimes I_{3}\right) \tilde{Q} u=\sum_{i}\left(P^{-1} E_{i} \otimes M\right)\left(\left(I_{N} \otimes L\right) u_{i}-g_{i}\right)
$$

where $L$ is an operator that describes the boundary conditions in the extended domain, $I_{3}$ and $I_{N}$ are 3-by-3 and N-by-N identity matrices, respectively. The index $i$ runs over the grid points in the extended domain close to the boundary and the matrix $M$ is a 3-by-3 penalty matrix to be determined such that the formulation remains stable. The operator $L$ can be chosen arbitrary, as long as one has the required knowledge of the solution in the extended domain. Here, we will consider two cases:

- the boundary conditions in (3) are known in a extended domain close to the inflow boundary.

- the boundary conditions in (7) are known in the extended domain close to the outflow boundary.

The determine the penalty matrix $M$, we multiple (13) with $u^{T}\left(P \otimes I_{3}\right)$ from the left and get (let $\left.g_{i}=0\right)$

$$
\|u\|_{t}^{2} \leq \sum_{i} u^{T}\left(E_{i} \otimes M L\right) u
$$

and we have stability if $M L \leq 0$. Stability is thus preserved by choosing $M=\nu_{i} L^{T}$ with $\nu_{i} \leq 0$, which ensures bounded energy in (14). 


\section{Results}

Numerical experiments has been performed for the one-dimensional case discussed above. All boundary data are zero and the initial condition $u(0, t)=[1,1,1]^{T}$ is used. Additional penalties are added in the manner described above with $M=-L^{T}$ at all points in the extended domain. The parameters in (1) are put to

$$
\gamma=1.4, \quad \bar{c}=2 \bar{v}=2, \quad \bar{\lambda}=-2 / 3 \quad \bar{\mu}=1, \quad \operatorname{Pr}=0.7, \quad \bar{k}=1, \quad \bar{\rho}=1 .
$$

The numerical experiments were performed for the linearized Euler equations, obtained by putting $\epsilon=0$ in (1) and the linearized Navier-Stokes equations with $\epsilon=0.01$ and $\epsilon=0.1$. In Figure 1, results from $\epsilon=0$ with additional penalties at the grid points closest to the inflow boundary are shown. One can see an increase of the rate of convergence to steady-state, but the effect vanish quickly as the mesh is refined. Increasing the number of penalties as the mesh is refined only partly remedies this effect.

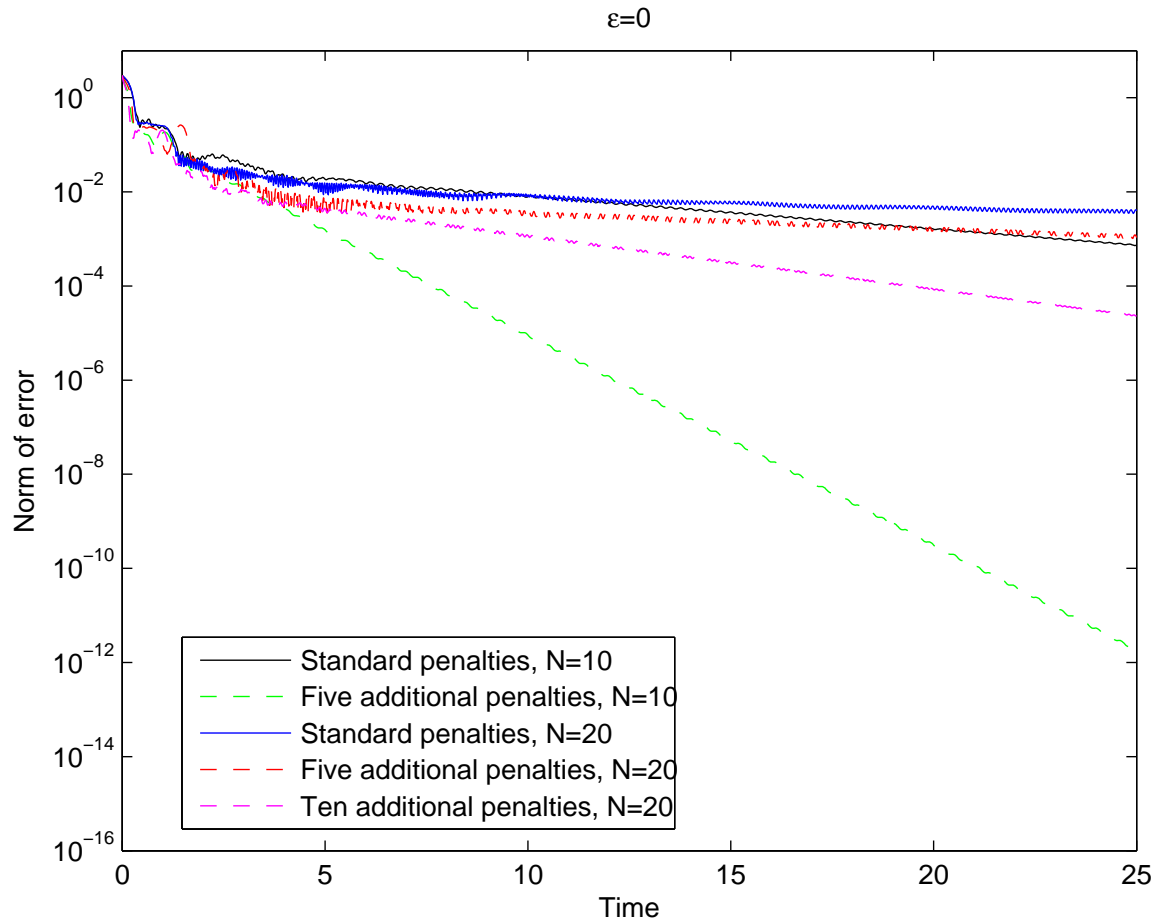

Figure 1. Error as a function of time with and without multiple penalties close to the inflow boundary. $\epsilon=0$

When the multiple penalties are added close to the outflow boundary, the effect decrease for the case $\epsilon=0$, as seen in Figure 2. In this case, the technique have a small effect on the rate of convergence. As the mesh is refined, the effect of multiple penalties vanish.

The effect of adding multiple penalties is more pronounced for $\epsilon \neq 0$. In Figure 3 and $4, \epsilon=0.01$ and multiple penalties are added close to the inflow and outflow boundary, respectively. The effect is similar for both kind of multiple penalties, but the technique applied to the inflow boundary conditions gives better results. In contrary to to pure hyperbolic case, the effect of multiple penalties remains, or is even slightly improved, as the mesh is refined.

Figure 5 and 6 shows the results with $\epsilon=0.1$ and multiple penalties added close to the inflow and outflow, respectively. The results are similar to the case $\epsilon=0.01$, with an even faster convergence to steady-state, as expected. The effect does not vanish when the mesh is refined. 
$\varepsilon=0$

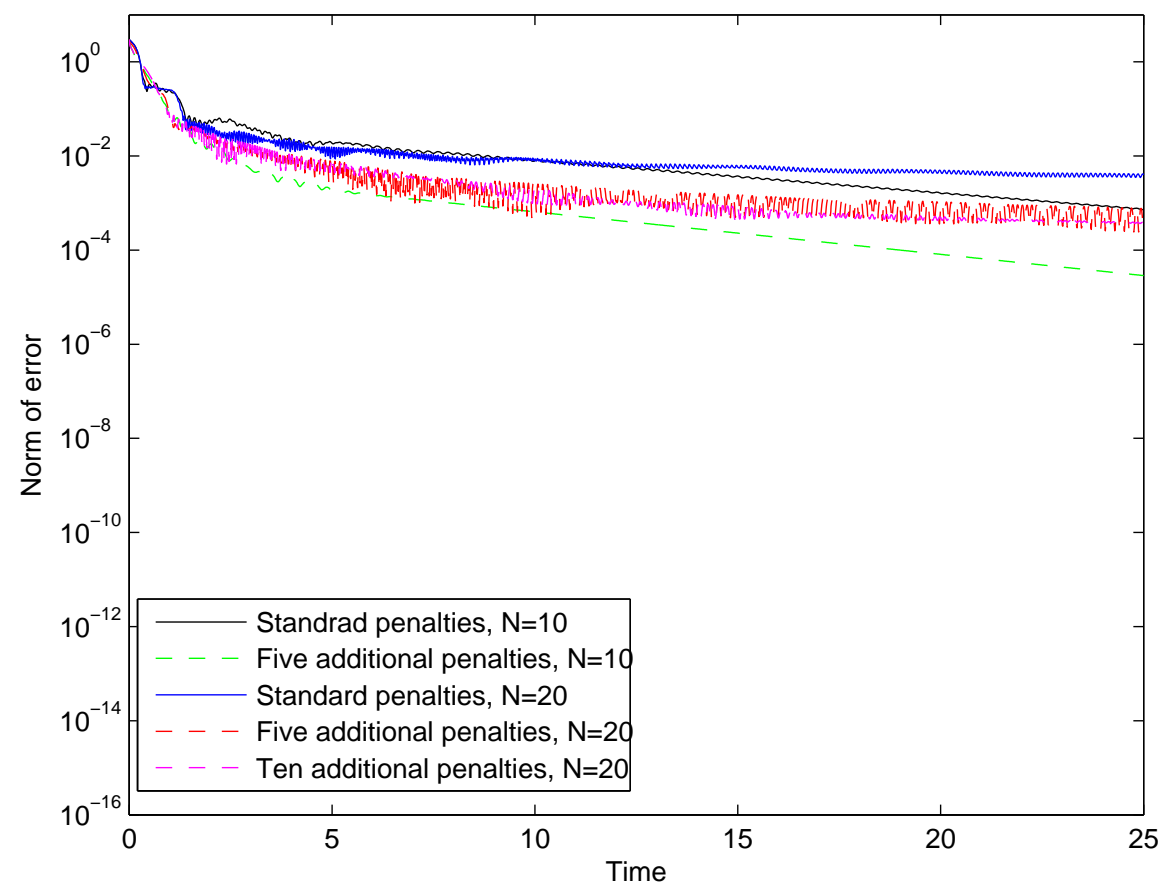

Figure 2. Error as a function of time with and without multiple penalties close to the outflow boundary. Note that the result with ten penalties and $N=20$ almost coincide with the result with five penalties and $N=20$. $\epsilon=0$

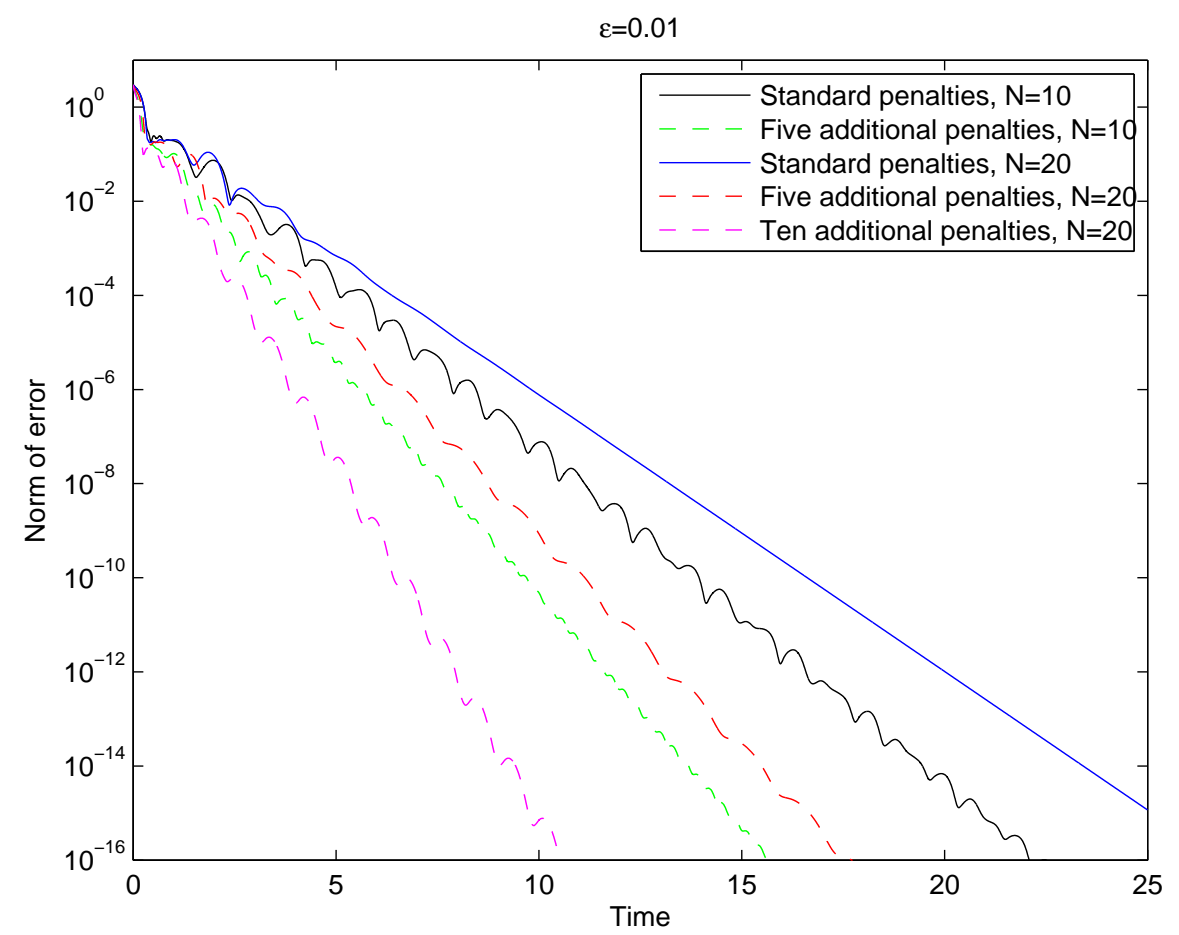

Figure 3. Error as a function of time with and without multiple penalties close to the inflow boundary. $\epsilon=0.01$ 


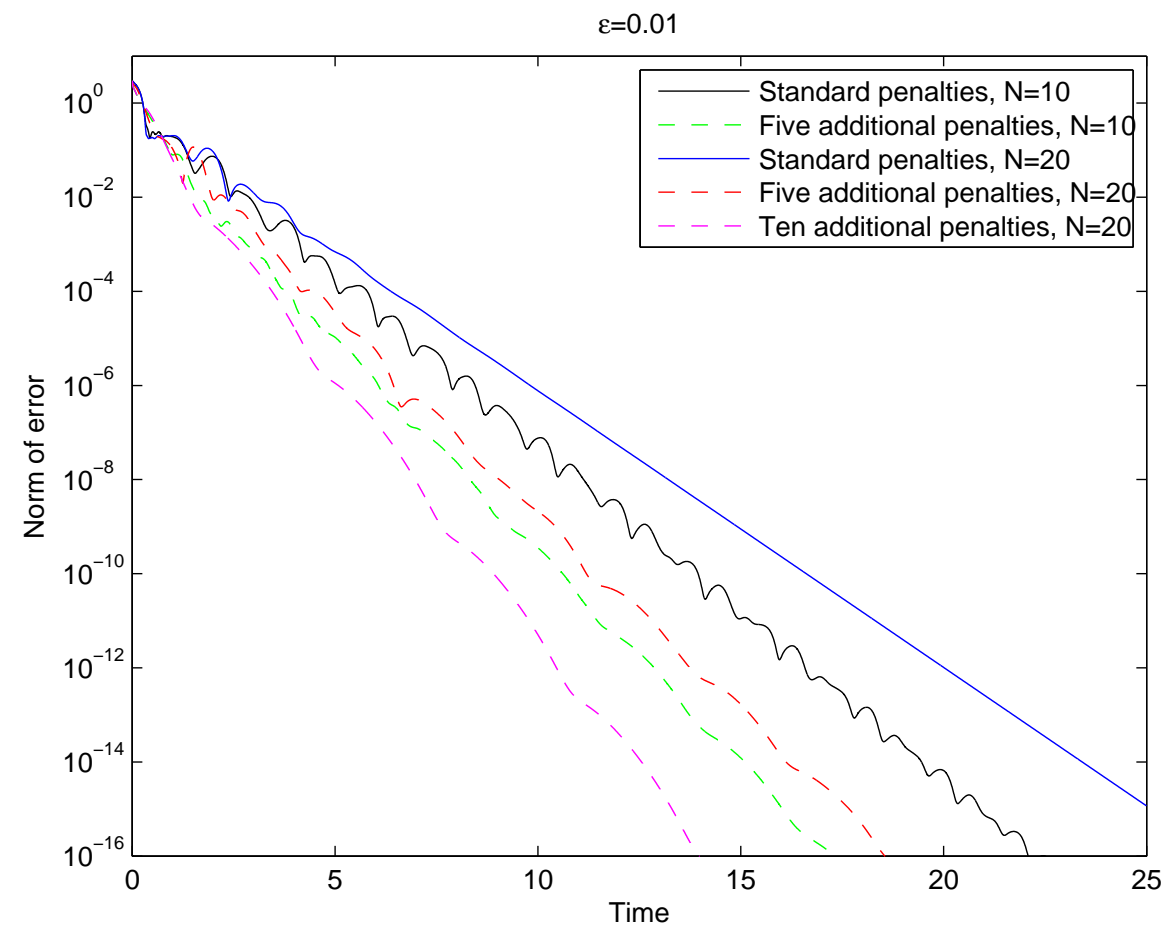

Figure 4. Error as a function of time with and without multiple penalties close to the outflow boundary. $\epsilon=0.01$

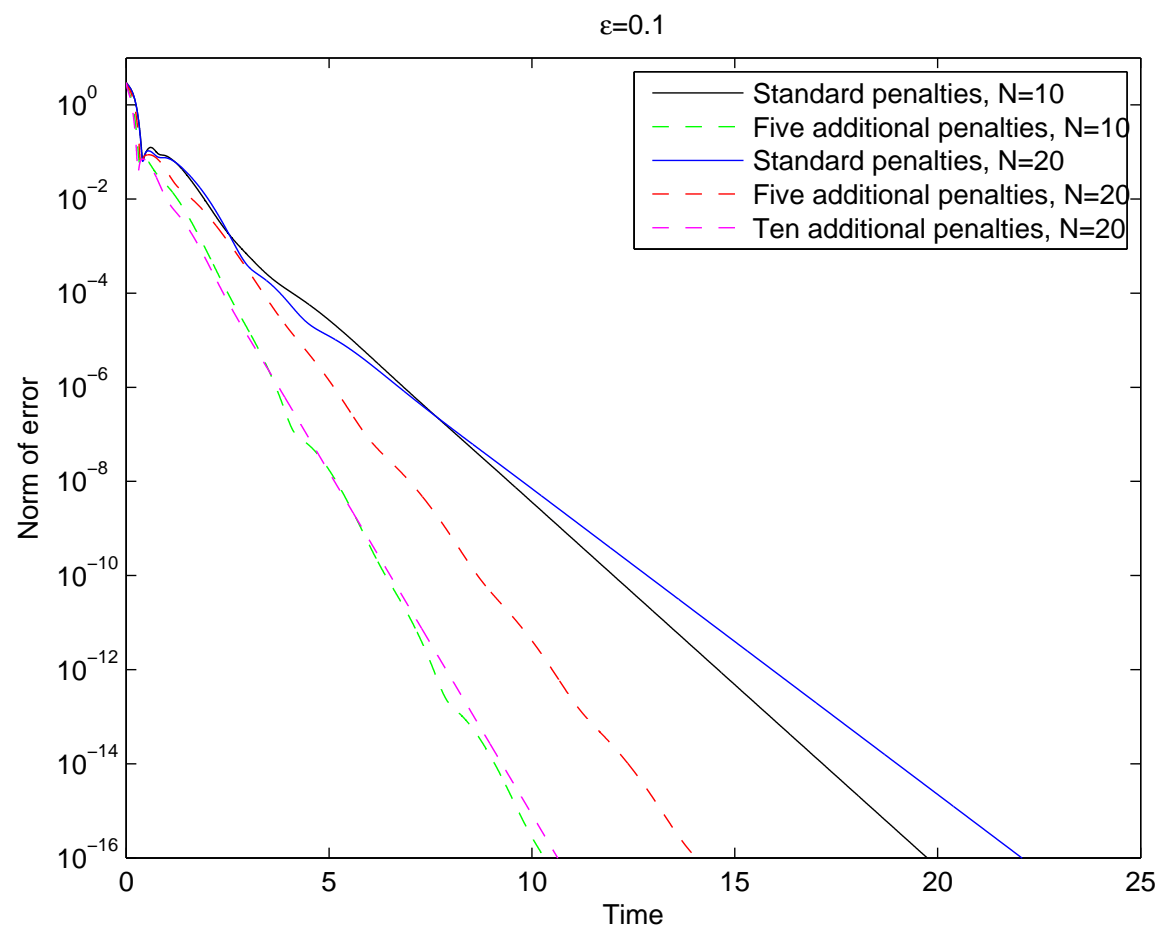

Figure 5. Error as a function of time with and without multiple penalties close to the inflow boundary. $\epsilon=0.1$ 


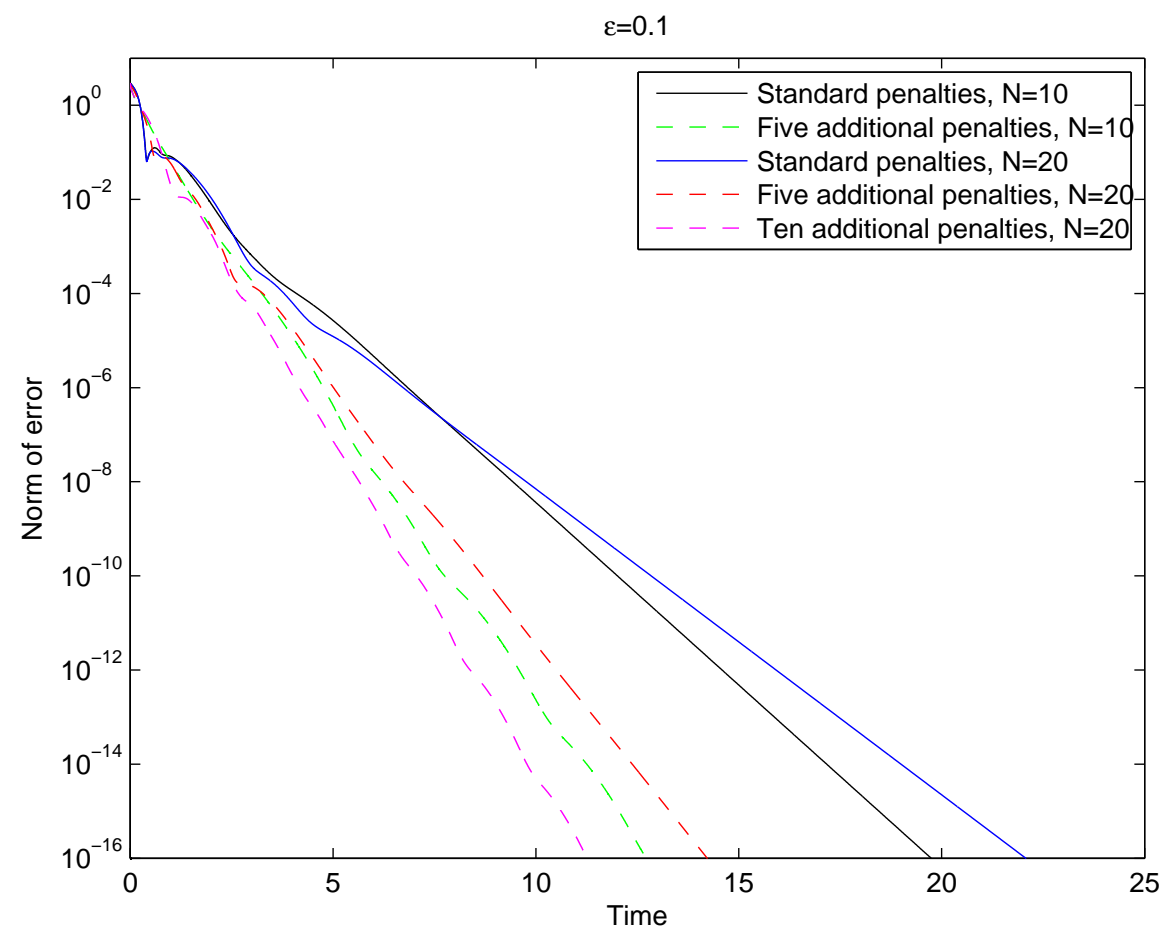

Figure 6. Error as a function of time with and without multiple penalties close to the outflow boundary. $\epsilon=0.1$

\section{Conclusions and future work}

The multiple penalties technique has been applied close to the boundaries such that stability is preserved by appropriate choices of penalty matrices. We have demonstrated how this is done for one-dimensional hyperbolic and parabolic systems and how the rate of convergence to steady-state increases.

The multiple penalties technique have a positive impact on the rate of convergence to steady state for all values of $\epsilon$, but the effect is more pronounced for $\epsilon>0$.

The method for obtaining stability described in (13) and (14) can easily be generalized to multiple space dimensions using the SBP operators.

As observed in Figures 1-6, the technique has a small impact on the rate of convergence for inviscid problem, especially for the finer mesh. For the viscous problem, the effect is more pronounced and the effect increases or remains constant as the mesh is refined.

Future work will include a study on how to optimize the convergence rate to steady-state. We will also investigate how to use the multiple penalty technique to obtain less reflection from the boundaries.

\section{References}

${ }^{1}$ B. Strand. Summation by parts for finite difference approximations for $\frac{d}{d x}$. Journal of Computational Physics,110:4767,1993 .

${ }^{2}$ K. Mattson, J. Nordström. Summation by parts for finite difference approximations of second order derivatives. Journal of Computational Physics,199:503-540,2004.

${ }^{3}$ M.H Carpenter, J. Nordström and D. Gottlieb. A stable and conservative interface treatment of arbitrary spatial accuracy. Journal of Computational Physics,148:341-365,1999

${ }^{4}$ M.H Carpenter, D. Gottlieb and S. Aberbanel. Time-stable boundary conditions for finite-difference schemes solving hyperbolic systems: methodology and application to high-order compact schemes. Journal of Computational Physics 111(2):220236,1994

${ }^{5}$ Q. Abbas and Jan Nordström. A weak boundary procedure for high order finite difference approximations of hyperbolic problems. Technical report. Department of Information Technology, Uppsala Univeristy, ISSN 1404-3203, 2011-019, 2011 
${ }^{6} \mathrm{~J}$-P. Berenger. A perfectly matched layer for the absorption of electromagnetic waves. Journal of Computational Physics, 114: $185-200,1994$

${ }^{7}$ D.J Bodony. Analysis of sponge zones for computational fluid mechanics. Journal of Computational Physics,212(2) 681702,2006

${ }^{8} \mathrm{~S}$. Aberbanel and D. Gottlieb. Optimal time splitting for two- and three-dimensional Navier-Stokes equations with mixed derivatives. Journal of Computational Physics,41:1-33, 1981

${ }^{9}$ J. Lindström and J. Nordström. A stable and high-order conjugate heat transfer problem. Journal of Computational Physics,229:5440-5456, 2012 